\title{
RR LYRAE STARS IN THE MAGELLANIC CLOUDS
}

\author{
ALISTAIR R WALKER \\ Cerro Tololo Inter-American Observatory, \\ Casilla 603, La Serena, Chile.
}

\begin{abstract}
RR Lyrae variables are a component of the oldest stellar populations. In the Magellanic Clouds they occur in a few clusters and in the general field. Recent observations have concentrated on obtaining accurate photometry for the cluster variables and the evolved stars of their parent clusters; these data have been used to determine abundances, distances and ages. Results from this work are reviewed.
\end{abstract}

\section{Introduction}

RR Lyraes were first discovered in the Magellanic Clouds (MC) near NGC 121 by Thackeray (1951); with $\langle\mathrm{B}\rangle=19-20$ they were a magnitude fainter than expected and at the time this provided the best evidence that the distance scale was incorrect by a factor two. This highlights one of the uses of RR Lyraes i.e. as distance indicators. RR Lyraes underpin the whole Population II distance scale. With $4 \mathrm{~m}$ class telescopes they can be measured throughout the local group. Only in the MC, and in particular the LMC, can accurate comparisons be made between various distance indicators. The occurrence of RR Lyraes in several LMC clusters (NGC 1466, 1786, 1835, 1841, 2210, 2257, GLC 0435-59) means that individual distances can be calculated for each of the clusters.

RR Lyraes indicate the existence of a population greater than $10 \mathrm{Gyr}$ old, assuming that $[\mathrm{CNO} / \mathrm{Fe}]$ is not radically different from that for RR Lyraes in galactic globular clusters. Their abundances $[\mathrm{Ca} / \mathrm{H}]$ can be measured. Therefore, in the MC clouds, RR Lyraes are important in discussions of the metal enrichment history. The distribution of field RR Lyraes can give clues as to the dynamical evolution of the MC and their past interactions with the Galaxy. Evolution of stars in the corehelium burning phase is one of the least understood stages of stellar evolution. Pulsation properties of the RR Lyraes may clarify this aspect, aided by the stars all being at constant distance, to a first approximation. In this review I will concentrate on work published since the last comprehensive review (Graham 1984) of the subject.

\section{Surveys for RR Lyraes}

There have been no published new surveys for MC field RR Lyraes. M. Hazen and J. Nemec are studying a 50 arc min. diameter field around NGC 2210. Some 50 field variables have been found, and the stars have now had periods determined. T. Kinman and L. Stryker are working on three fields near the clusters NGC 1466, NGC 1841 and GLC 0435-59 (Reticulum). These observations 
are all based on CTIO $4 \mathrm{~m}$ PF plates. Horace Smith is searching for variable stars on CTIO $1.5 \mathrm{~m}$ plates, exposed by John Graham, of the NGC 361 region in the SMC. Reid and Freedman (1990) have identified 3700 variables from UK Schmidt plates covering $36 \mathrm{deg}^{2}$ centered on the LMC. Some 500 of the variables are possible RR Lyraes; analysis of more plates, and spectroscopy, are planned. Thus the only intensively studied regions, excluding work in progress mentioned above, are a 50 arc min. diameter field centered on NGC 2257 (Nemec et al. 1985b) and 1 x $1.3 \mathrm{deg}$. fields around NGC 121 and 1783 (Graham 1975, 1977). The latter two surveys are liable to be seriously incomplete for RRc stars.

Graham and Nemec (1984) report on a photographic survey of MC clusters for RR Lyraes; with their new discoveries RR Lyraes are known in eight MC clusters. J. Nemec has found more variables in NGC 2210. Gratton and Ortolani (1987) find that the cluster GLC 0435-59 is associated with the LMC, earlier, Demers and Kunkel (1976) discovered RR Lyraes in this cluster. Walker (1989a) reports on a survey for RR Lyraes in five SMC clusters, which involved blinking $U$ and B CCD frames of the clusters and also the preparation of color magnitude diagrams (CMDs). The clusters surveyed were NGC 121, Lindsay 1, Kron 3, Kron 7 and Kron 44. The NGC 121 RR Lyraes were rediscovered, but none were found in the other clusters. Since NGC 121 has age $12 \pm 2$ Gyr (Stryker et al. 1985) and Lindsay 1, the next oldest SMC cluster, $10 \pm 2$ Gyr (Olszewski et al. 1987), this defines the minimum age at which $R R$ Lyraes appear at $\approx 11 \mathrm{Gyr}$.

\section{RR Lyraes in Magellanic Cloud Clusters}

There are only a handful of MC clusters which contain RR Lyraes, and are therefore of an age comparable with those of galactic globular clusters. Hodge 11, which from all available indications (Stryker et al. 1984) is old, does not contain RR Lyraes In this case the reason appears to be that all the horizontal branch (HB) stars lie on the blue side of the instability strip.

The earlier photographic photometry of RR Lyraes in clusters must be corrected for radial errors, and in the MC the variables are faint enough that calibrating sequences for both cluster and field variables are generally photographic extensions of photoelectric sequences and thus are also suspect. In fact, checks have shown that at least some of this work is of remarkable accuracy, in particular the Graham (1977) NGC 1783 field, checked by Blanco and Blanco (1986), and the Nemec et al. (1985b) work on NGC 2257. CCD photometry has allowed great improvements in the photometry for these stars, and in addition has permitted measurement in the more crowded central cluster regions. Multi-color light curves allow determination of $[\mathrm{Fe} / \mathrm{H}]$ from Period-Amplitude plots (Sandage 1982) and reddening via the color at minimum light (Sturch 1966). The CCD photometry has also enabled study of the cluster CMDs.

\subsection{NGC 121}

NGC 121 is the oldest cluster in the SMC. The CMD of Stryker et al. (1985) gives an age of 12 \pm 2 Gyr. Unpublished photometry (A. Walker), fitted with the Vandenberg and Bell (1985) isochrones scaled to a $\mathrm{HB}$ magnitude of $\mathrm{V}_{0}=19.46$ (Walker and Mack 1988) and assuming $\left\langle\mathrm{M}_{\mathrm{V}}(\mathrm{RR})\right\rangle=0.6$, suggests that NGC 121 has age 12-14 Gyr, and the surrounding field may be equally old. However there are some interesting anomalies in the CMD and a comprehensive study of this cluster and the surrounding field is clearly warranted. The metal abundance of NGC 121 is near $[\mathrm{Fe} / \mathrm{H}]=-1.4$, according to Stryker et al. (1985), and the Period-Amplitude relation for the RR Lyraes agrees with this value or one slightly more metal poor. 


\subsection{LMC CLUSTERS}

NGC 1841 (Walker 1990), GLC 0435-59 (Gratton and Ortolani 1987), NGC 2257 (Stryker 1983) and NGC 121 (Stryker et al. 1985) all have CMDs reaching fainter than the MS turn-off. There is little doubt that NGC 1841 and GLC $0435-59$ are as old as galactic globulars such as M92(i.e.16-18 Gyr when Vandenberg and Bell (1985) isochrones are fitted). NGC 2257 is probably equally as old, although the (photographic) photometry has more scatter than the more recent CCD work. There thus appears to be conclusive evidence that at least some of the oldest LMC clusters are as old as those in the Galaxy, and therefore contain RR Lyraes that are not younger than their Galactic counterparts.

Butler et al. (1982) found that six RR Lyraes in the NGC 1783 field (Graham 1977) have mean $[\mathrm{FE} / \mathrm{H}]=-1.6 \pm 0.1$ (Zinn and West (1984) zero-point), while from the Period-Amplitude relation for nine stars in the NGC 2257 field, $[\mathrm{Fe} / \mathrm{H}]=-1.8 \pm 0.1$, identical to that for the NGC 2257 variables themselves. Period-Amplitude plots for the RR Lyraes in NGC 1786 and 1841 show that these clusters are very metal poor, $[\mathrm{Fe} / \mathrm{H}] \approx-2.2$. Therefore, in the $\mathrm{LMC}$, the field RR Lyraes are more metal rich than are some of the oldest clusters.

The RR Lyrae data for NGC 2257 (Nemec et al. 1985a, 1985b; Walker 1989b) are now very extensive, and include the only available measurements of period changes for extra-galactic RR Lyraes. Only one star, out of 37 , appears to have had an abrupt change in period. Significantly more (9 out of $16,56 \%$ ) RRc stars have constant periods than do RRab stars (5 out of 13,38\%). CCD frames should be taken at regular (e.g. yearly) intervals in order to continue to track the period changes.

\section{Field - Cluster Comparison}

With presently available data this is only possible in reasonable detail near NGC 2257 (Nemec et al. 1985b, Walker 1989b). There are selection effects involved when comparing field and cluster RR Lyraes, for volume-limited field star work will tend to discover RR Lyraes in their longest-lived evolutionary phase, i.e. close to the ZAHB, while for very metal-poor clusters the RR Lyraes may well be evolving away from the ZAHB and consequently could be at least 0.2 mag brighter than the field stars (Lee et al. 1990). The NGC 2257 field RR Lyraes are in fact in the mean almost 0.2 mag fainter than the cluster variables, even though their mean metal abundance is identical (Walker 1989b). If identical $M_{V}(R R)$ is assumed then the difference in distance between NGC 2257 field and cluster amounts to $3.7 \mathrm{kpc}$. However the alternative supposition, that the evolutionary state of the two sets of stars is different, cannot be ruled out. In this context it is very important to obtain more observations for the possible RRd stars in NGC 2257, and to search for RRd stars amongst the field stars, in order to allow masses for the field and cluster variables to be compared. Fourier decomposition of high quality light curves should also be attempted.

The (CCD) mean $\langle\mathrm{B}\rangle$ and $\langle\mathrm{V}\rangle$ magnitudes for the NGC 121 variables is identical to the (photographic) mean $\langle\mathrm{B}\rangle$ and $\langle\mathrm{V}\rangle$ for the field RR Lyraes near NGC 121. This result is of considerable interest, if confirmed. Butler et al. (1982) found four SMC field RR Lyraes to have mean $[\mathrm{Fe} / \mathrm{H}]=-2.0 \pm 0.2$ (Zinn and West (1984) scale). Unlike in the LMC, the field SMC field RR Lyraes appear more metal poor than the oldest clusters.

Abundances for the field RR Lyraes will be of critical importance in unravelling the early history of the MC. Period-Amplitude plots can be used to determine $[\mathrm{Fe} / \mathrm{H}]$, although this method appears 
to work best when a number of results can be averaged, and indeed should be strictly applied to ZAHB stars only. In any case, the phase uncertainty that will have developed over the 20 years since the Graham $(1975,1977)$ studies will necessitate new photometry for these stars, prior to spectroscopy at minimun light.

\section{RR Lyrae Absolute Magnitudes}

The question of the absolute magnitude of the Horizontal Branch, and its variation with abundance, is important and controversial. We will proceed by adopting a distance to the LMC, choosing a model for the geometry of the LMC, and then finding a mean $M_{V}(R R)$ for the cluster RR Lyraes. Finally we will see whether the data can address the question of the magnitude-metallicity relation.

The LMC distance modulus itself has been highly controversial throughout the past decade, from the time when the first CCD CMDs of LMC clusters were found to be best fitted with a distance modulus of $18.2-18.3 \mathrm{mag}$ whereas the then current Cepheid modulus was 18.7 mag. Revision of the temperature-color scale for the cluster isochrones (Vandenberg and Poll 1989) has increased the cluster moduli by $\approx 0.2 \mathrm{mag}$, and revised distances for the galactic clusters containing Cepheids (Feast and Walker 1987) has decreased the Cepheid modulus by $\approx 0.25 \mathrm{mag}$. We will assume here a Cepheid modulus of $18.47 \mathrm{mag}$, and note that this has an external error of $\pm 0.15 \mathrm{mag}$ of which \pm 0.07 arises from the uncertainty in the modulus of the Pleiades, whose distance in turn is derived directly from the trigonometrical parallaxes of early type stars. The Cepheid modulus for the SMC is 18.78 mag. Feast (1989) reviews the depth structure of the MC; the great line-of-sight depth of the SMC makes the assignation of distances for individual SMC objects particularly hazardous.

We will adopt the Freeman et al. (1983) "old cluster" model for the LMC. This then defines the distance modulus for each cluster, and $M_{V}(R R)$ follows, after corrections for reddening have been made. The results are summarized in Table 1 . Giving the GLC $0435-59$ measurements half weight in view of the fact that the RR Lyrae magnitude is a single-epoch mean (Gratton and Ortolani 1987), we find $M_{V}(R R)=0.47 \pm 0.03$ (se) where the error takes account of the scatter in the individual measurements only, and does not include errors in the photometry, reddening

Table 1. Old LMC clusters with CCD photometry. Cluster moduli are calculated from a model with inclination $27^{\circ}$, nodal position angle $44^{\circ}$, and LMC modulus $18.47 \mathrm{mag}$.

\begin{tabular}{|c|c|c|c|c|c|c|c|}
\hline Cluster & $\mathrm{PA}^{\circ}$ & radius $^{\circ}$ & mod. & $<\mathrm{V}(\mathrm{RR})\rangle$ & $\mathrm{M}_{\mathrm{V}}(\mathrm{RR})$ & {$[\mathrm{Fe} / \mathrm{H}]$} & $P_{A B}$ \\
\hline 1786 & 304 & 2.5 & 18.51 & 19.04 & 0.53 & -2.3 & 0.677 \\
\hline 1841 & 183 & 14.8 & 18.29 & 18.73 & 0.44 & -2.2 & 0.676 \\
\hline 2210 & 86 & 4.5 & 18.42 & 18.94 & 0.52 & -1.9 & 0.602 \\
\hline 2257 & 61 & 8.5 & 18.43 & 18.91 & 0.48 & -1.8 & 0.574 \\
\hline Ret. & 325 & 10.1 & 18.64 & 19.01 & 0.37 & -2.0 & \\
\hline
\end{tabular}

or LMC distance. We note that this result is not strongly dependent on the adopted LMC model, for if all the clusters are assumed to have a distance modulus of $18.47 \mathrm{mag}$, then $\mathrm{M}_{\mathrm{V}}(\mathrm{RR})=0.45$ $\pm 0.05(\mathrm{se})$. The mean cluster abundance is $[\mathrm{Fe} / \mathrm{H}]=-2.0$.

The small range of abundances amongst the old LMC clusters makes the search for a magnitudemetallicity relation, using the clusters alone, impossible given the errors. Two other methods are available. The first is to compare the differential LMC-SMC distances for both Cepheids and RR 
Lyraes. If an incorrect RR Lyrae magnitude - metallicity relation is used then these distances will be different. The differential Cepheid modulus is $0.31 \mathrm{mag}$, while the difference in RR Lyrae mean magnitudes is $(19.46 \pm 0.07)-(18.94 \pm 0.03)=0.52 \pm 0.08$. The difference in metal abundance is $0.6 \pm 0.2 \mathrm{dex}$, therefore $\delta \mathrm{M}_{\mathrm{V}} / \delta[\mathrm{Fe} / \mathrm{H}]=0.35 \pm 0.2$. Additional sources of error may arise from the assumption that NGC 121 is at the mean distance of the SMC Cepheids. The second method is to compare the absolute magnitude for the LMC RR Lyraes with the statistical parallax results (e.g. Barnes and Hawley (1986)). These results give $M_{V}(R R)=0.70 \pm 0.15$ for a sample with mean $[\mathrm{Fe} / \mathrm{H}] \approx-1.2$ (Butler 1975, Zinn and West 1984), therefore $\delta \mathrm{M}_{\mathrm{V}} / \delta[\mathrm{Fe} / \mathrm{H}]=0.3 \pm 0.2$. Unfortunately, neither method has small enough errors to determine the ratio accurately. However it seems clear that the ratio is probably not zero.

\section{Magellanic Cloud Structure}

It has been long known (Graham 1973) that the magnitude dispersion amongst LMC field RR Lyraes is smaller than expected if the stars have a spheroidal distribution, and this is confirmed by the more recent work in the NGC 2257 region. In addition there appears to be no strong central concentration. Study of the field RR Lyraes, in several lines-of-sight, in both MC would pay handsome dividends in the form of elucidating MC structure as delineated by its oldest population. That this project has not yet been attempted with CCDs is no doubt due to the low spatial density of the RR Lyraes and the small size of current CCD detectors. The latter technical limitation should be removed in the near future, although the project will still require a considerable investment in telescope time, since even a 20 arc min square LMC field is expected to contain only $\approx 10 \mathrm{RR}$ Lyraes.

All that can be done at present is to use the clusters with HB CCD photometry as probes of the LMC structure, in the following way. If we assume that the clusters do lie in a plane (note that to first order all LMC constituents have a planar distribution (Feast 1989)) then we can alter the position angle and inclination of the plane and see what effect it has on $M_{V}(R R)$. With two variables and only five constraints the test of the model is somewhat crude (observations of the RR Lyraes in NGC 1466 and 1835 are scheduled). Some examples are given in Table 2, where the inclinations cover the range of values suggested at various times, while the two values for the position angle are the "young" and "old" solutions from Freeman et al. (1983). The smallest standard deviation is in fact that for the inclination and position angle derived (kinematically) by Freeman et al. (1983) for the older LMC clusters.

Table 2. The standard deviation (mag) of the mean RR Lyrae $M_{V}$ for five clusters are derived for a few values of LMC inclination and position angle.

\begin{tabular}{lll}
\hline Incl $^{\circ}$ & PA $^{\circ}$ & $\sigma$ \\
\hline 27 & 44 & 0.07 \\
45 & 44 & 0.18 \\
27 & 1 & 0.14 \\
45 & 1 & 0.21 \\
0 & & 0.12 \\
\hline
\end{tabular}

\section{Future Work}

In the past five years observations of RR Lyraes in the MC clusters have been valuable in the 
discussion of questions involving the distance scale, age and structure of the MC. There has been little published work on the field RR Lyraes, yet these are of equal or greater importance in clarifying the structural and chemical evolution of the MC more than $10 \mathrm{Gyr}$ ago. It is to be hoped over the next few years that photometry and spectroscopy for significant numbers of the field variables will become available, in order to help to unravel the early history of the MC.

\section{References}

Barnes, T.G., and Hawley, S.L. (1986). Astrophys. J. 307, L9.

Blanco, V.M., and Blanco, B.M. (1986). Publ. Astron. Soc..Pac. 98, 1162.

Butler, D. (1975). Astrophys. J. 200, 68.

Butler, D., Demarque, P., and Smith, H.A. (1982). Astrophys. J. 257, 592.

Demers, S., and Kunkel, W.E. (1976). Astrophys. J. 208, 932.

Feast, M.W. (1989). In "Recent Developments of Magellanic Cloud Research", edited by K.S. de Boer, F. Spite, and G. Stasinska (Observatoire de Paris, Paris), p. 75.

Feast, M.W., and Walker, A.R. (1987). Annu. Rev. Astron. Astrophys. 25, 345.

Freeman, K.C., Illingworth, G.D., and Oemler, A. (1983). Astrophys. J. 272, 488.

Graham, J.A. (1973). In "Variable Stars in Globular Clusters and Related Systems", edited by J.D. Fernie (Reidel, Dordrecht), p. 207.

Graham, J.A. (1975). Publ. Astron. Soc. Pac. 87, 641.

Graham, J.A. (1977). Publ. Astron. Soc. Pac. 89, 425.

Graham, J.A. (1984). In "IAU Symposium No. 108", edited by S. van den Bergh and K.S. de Boer (Reidel, Dordrecht), p. 207

Graham, J.A., and Nemec, J.M. (1984). In "IAU Symposium No. 108", edited by S. van den Bergh and K.S. de Boer (Reidel, Dordrecht), p. 37.

Gratton, R.G., and Ortolani, S. (1987). Astron. Astrophys. Suppl. 71, 131.

Lee, Y-W., Demarque, P., and Zinn, R.J. (1990). Astrophys. J., 350, 155.

Nemec, J.M., Hazen-Liller, M., and Hesser, J.E. (1985a). Astrophys. J. Suppl. 57, 329.

Nemec, J.M., Hesser, J.E., and Ugarte, P. (1985b). Astrophys. J. Suppl. 57, 287.

Olszewski, E.W., Schommer, R.A., and Aaronson, M. (1987). Astron. J. 93, 565.

Reid, N., and Freedman, W.L. (1990). Pulsation-Evolution Conf., Bologna. Astron. Soc. Pac. Conf. Ser., in press.

Sandage, A. (1982). Astrophys. J. 252, 553.

Stryker, L.L. (1983). Astrophys. J. 266, 82.

Stryker, L.L, Nemec, J.M., Hesser, J.E., and McClure, R.D., (1984). In "IAU Symposium No. 108", edited by S. van den Bergh and K.S. de Boer (Reidel, Dordrecht), p. 43.

Stryker, L.L., da Costa, G.S., and Mould, J.R. (1985). Astrophys. J. 298, 544.

Sturch, C.R. (1966). Astrophys. J. 143, 774.

Thackeray, A.D. (1951). The Observatory 71, 219.

Vandenberg, D.A., and Bell, R.A. (1985). Astrophys. J. Suppl. 58, 561.

Vandenberg, D.A., and Poll, H.E. (1989). Astron. J. 98, 1451.

Walker, A.R. (1989a). Publ. Astron. Soc. Pac. 101, 570.

Walker, A.R. (1989b). Astron. J. 98, 2086.

Walker, A.R. (1990). Astron. J. in press.

Walker, A.R., and Mack, P. (1988). Astron. J., 96, 872.

Zinn, R., and West, M.J. (1984). Astrophys. J. Suppl. 55, 45. 